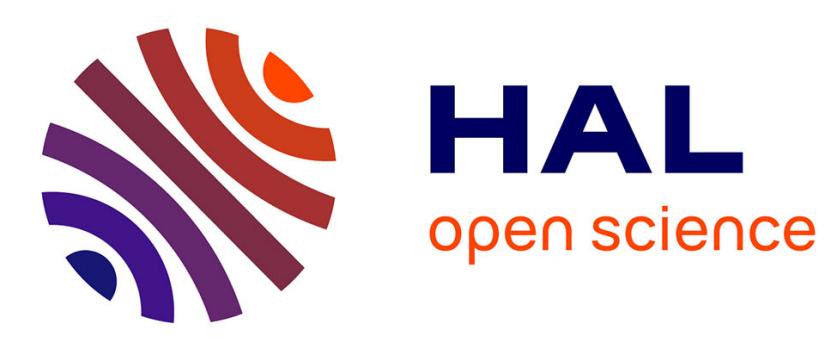

\title{
Game Control Problem for Systems of Distributed Equations
}

Vyacheslav Maksimov

\section{To cite this version:}

Vyacheslav Maksimov. Game Control Problem for Systems of Distributed Equations. 27th IFIP Conference on System Modeling and Optimization (CSMO), Jun 2015, Sophia Antipolis, France. pp.360-369, 10.1007/978-3-319-55795-3_34. hal-01626906

\section{HAL Id: hal-01626906 https://hal.inria.fr/hal-01626906}

Submitted on 31 Oct 2017

HAL is a multi-disciplinary open access archive for the deposit and dissemination of scientific research documents, whether they are published or not. The documents may come from teaching and research institutions in France or abroad, or from public or private research centers.
L'archive ouverte pluridisciplinaire HAL, est destinée au dépôt et à la diffusion de documents scientifiques de niveau recherche, publiés ou non, émanant des établissements d'enseignement et de recherche français ou étrangers, des laboratoires publics ou privés. 


\title{
Game Control Problem for Systems of Distributed Equations
}

\author{
Vyacheslav Maksimov \\ Moscow State University, Moscow, \\ Institute of Mathematics and Mechanics of UB RAS, \\ Ekaterinburg, Russia
}

\begin{abstract}
We consider a game problem of guaranteed positional control for a distributed system described by the phase field equations under incomplete information on system's phase states. This problem is investigated from the viewpoint of the first player (the partner). For this player, a procedure for forming feedback controls is specified. This procedure is stable with respect to informational noises and computational errors and is based on the method of extremal shift and the method of stable sets from the theory of guaranteed positional control. It uses the idea of stable dynamical inversion of controlled systems.
\end{abstract}

Keywords: guaranteed control, phase field equations

\section{Introduction}

The control theory for distributed systems has been intensively developed in recent time as a part of mathematical control theory. At present, there exists a number of monographs devoted to control problems for distributed systems [1-3]. As a rule, the emphasis is on program control problems in the case when all system's parameters are precisely specified. Along with this, the investigation of control problems for systems with uncontrollable disturbances (the problems of game control) is also reasonable. Similar problems have been poorly investigated. In the early 70es, N.N.Krasovskii suggested an effective approach to solving game (guaranteed) control problems, which is based on the formalism of positional strategies. The detailed description of this approach for dynamical systems described by ordinary differential equations is given in [4]. The goal of the present work is to illustrate possibilities of this approach for investigating a game problem for systems described by the phase field equations.

We consider a system modeling the solidification process and governed by the phase field equations (introduced in [5])

$$
\begin{aligned}
& \frac{\partial}{\partial t} \psi+l \frac{\partial}{\partial t} \varphi=k \Delta_{L} \psi+B u-C v \quad \text { in } \quad Q=\Omega \times\left(t_{0}, \vartheta\right], \\
& \tau \frac{\partial}{\partial t} \varphi=\xi^{2} \Delta_{L} \varphi+g(\varphi)+\psi, \quad \vartheta=\text { const }<+\infty,
\end{aligned}
$$

\footnotetext{
* This work was supported by the Russian Science Foundation (project 14-01-00539).
} 
with the boundary condition $\frac{\partial}{\partial n} \psi=\frac{\partial}{\partial n} \varphi=0 \quad$ on $\quad \partial \Omega \times\left(t_{0}, \vartheta\right]$ and the initial condition $\psi\left(t_{0}\right)=\psi_{0}, \quad \varphi\left(t_{0}\right)=\varphi_{0} \quad$ in $\quad \Omega$. Here, $\Omega \subset \mathbb{R}^{n}$ is a bounded domain with the sufficiently smooth boundary $\partial \Omega, \Delta_{L}$ is the Laplace operator, $\partial / \partial n$ is the outward normal derivative, $\left(U,|\cdot|_{U}\right)$ and $\left(V,|\cdot|_{V}\right)$ are Banach spaces, $B \in \mathcal{L}(U ; H)$ and $C \in \mathcal{L}(V ; H)$ are linear continuous operators, $H=L_{2}(\Omega)$, and the function $g(z)$ is the derivative of a so-called potential $G(z)$. We assume that $g(z)=a z+b z^{2}-c z^{3}$.

Systems of form (1) have been investigated by many authors. In what follows, for the sake of simplicity, we assume that $k=\xi=\tau=c=1$. Further, we assume that the following conditions are fulfilled: (A1) the domain $\Omega \subset \mathbb{R}^{n}, n=2,3$, has the boundary of $C^{2}$-class; (A2) the coefficients $a$ and $b$ are elements of the space $L_{\infty}(T \times \Omega), T=\left[t_{0}, \vartheta\right]$, and $\operatorname{vrai} \sup c(t, \eta)>0$ for $(t, \eta) \in\left[t_{0}, \vartheta\right] \times \Omega ;(\mathrm{A} 3)$ the initial functions $\psi_{0}$ and $\varphi_{0}$ are such that $\left\{\psi_{0}, \varphi_{o}\right\} \in \mathcal{R}=\left\{\psi, \varphi \in W_{\infty}^{2}(\Omega)\right.$ : $\frac{\partial}{\partial n} \psi=\frac{\partial}{\partial n} \varphi=0 \quad$ on $\left.\quad \partial \Omega\right\}$.

Introduce the notation: $W_{p}^{2,1}(Q)=\left\{u \mid u, \frac{\partial u}{\partial \eta_{i}}, \frac{\partial^{2} u}{\partial \eta_{i} \partial \eta_{j}}, \frac{\partial u}{\partial t} \in L^{p}(Q)\right\}$ for $p \in$ $[1, \infty)$ is the standard Sobolev space with the norm $\|u\|_{W_{p}^{2,1}(Q)} ;(\cdot, \cdot)_{H}$ and $|\cdot|_{H}$ are the scalar product and the norm in $H$, respectively. Let some initial state $x_{0}=\left\{\psi_{0}, \varphi_{0}\right\}$ and functions $u(\cdot) \in L_{\infty}(T ; U)$ and $v(\cdot) \in L_{\infty}(T ; V)$ be fixed. A solution of system (1), $x\left(\cdot ; t_{0}, x_{0}, u(\cdot), v(\cdot)\right)=\left\{\psi\left(\cdot ; t_{0}\right.\right.$, $\left.\left.\psi_{0}, u(\cdot), v(\cdot)\right), \varphi\left(\cdot ; t_{0}, \varphi_{0}, u(\cdot), v(\cdot)\right)\right\}$, is a unique function $x(\cdot)=x\left(\cdot ; t_{0}, x_{0}, u(\cdot)\right.$, $v(\cdot)) \in V_{T}^{(1)}=V_{1} \times V_{1}, \quad V_{1}=W_{2}^{2,1}(Q)$ satisfying relations (1). By virtue of the corresponding embedding theorem, without loss of generality, one can assume that the space $V_{T}^{(1)}$ is embedded into the space $C(T ; H \times H)$. Therefore, the element $x(t)=\{\psi(t), \varphi(t)\} \in H \times H$ is the phase state of system (1) at the time $t$. The following theorem takes place

Theorem 1. [6,p. 25, Assertion 5] Let conditions (A1)-(A3) be fulfilled. Then for any $u(\cdot) \in L_{\infty}(T ; U)$ and $v(\cdot) \in L_{\infty}(T ; V)$ there exists a unique solution of system (1).

The paper is devoted to the investigation of the game control problem. Let us give the informal formulation of this problem. Let a uniform net $\Delta=$ $\left\{\tau_{i}\right\}_{i=0}^{m}, \quad \tau_{i}=\tau_{i-1}+\delta, \quad \tau_{0}=t_{0}, \quad \tau_{m}=\vartheta$ with a diameter $\delta=\tau_{i}-\tau_{i-1}$ be fixed on a given time interval $T$. Let a solution of system (1) be unknown. At the times $\tau_{i} \in \Delta$, a part of the phase states $x\left(\tau_{i}\right)$ (namely $\phi\left(\tau_{i}\right)$ ) is inaccurately measured. The results of measurements $\xi_{i}^{h} \in H, i \in[1: m-1]$, satisfy the inequalities

$$
\left|\xi_{i}^{h}-\phi\left(\tau_{i}\right)\right|_{H} \leq h .
$$

Here, $h \in(0,1)$ is a level of informational noise. Let the following quality criterion be given: $I(x(\cdot), u(\cdot))=\sigma(x(\vartheta))+\int_{t_{0}}^{\vartheta} \chi(t, x(t), u(t)) d t$, where $\sigma: H \times H \rightarrow \mathbb{R}$ and $\chi: T \times H \times H \times U \rightarrow \mathbb{R}$ are given functions satisfying the local Lipschitz conditions. Let also a prescribed value of the criterion, number $I_{*}$, be fixed. The control problem under consideration consists in the following. There are two players-antagonists controlling system (1) by means of $u$ and $v$, respectively. 
One of them is called a partner; another, an opponent. Let $P \subset U$ and $E \subset V$ be given convex bounded and closed sets. The problem undertaken by the partner is as follows. It is necessary to construct a law (a strategy) for forming the control $u$ (with values from $P$ ) by the feedback principle (on the base of measurements of $\varphi\left(\tau_{i}\right)$ ) in order to provide the prescribed value of the quality criterion for any (unknown) realization $v=v(\cdot)$.

To form the control $u$ providing the solution of the problem, along with the information on the "part" of coordinates of the solution of system (1) (namely, on the values $\xi_{i}^{h}$ satisfying inequalities (2)), it is necessary to obtain some additional information on the coordinate $\psi(\cdot)$, which is missing. To get such a piece of information during the control process, it is reasonable, following the approach developed in [7-9], to introduce an auxiliary controlled system. This system is described by a parabolic equation (the form is specified below). The equation has an output $w_{*}(t), t \in T$, and an input $p^{h}(t), t \in T$. The input $p^{h}(\cdot)$ is some new auxiliary control; it should be formed by the feedback principle in such a way that $p^{h}(\cdot)$ "approximates" the unknown coordinate $\psi(\cdot)$ in the mean square metric. Thus, along with the block of forming the control in the real system (it is called an controller), we need to incorporate into the control contour one more block (it is called an identifier) allowing to reconstruct the missing coordinate $\psi(\cdot)$ in real time. Note that, in essence, the identifier block solves a dynamical inverse problem, namely, the problem of (approximate) reconstruction of the unknown coordinate $\psi(\cdot)$. In he recent time, the theory of inverse problems for distributed systems has been intensively developed. Among the latest investigations, it is possible to mark out the research [10].

\section{Problem statement}

Before passing to the problem formulation, we give some definitions. Furthermore, we denote by $u_{a, b}(\cdot)$ the function $u(t), t \in[a, b]$, considered as a whole. The symbol $P_{a, b}(\cdot)$ stands for the restriction of the set $P_{T}(\cdot)$ onto the segment $[a, b] \subset T$. Any strongly measurable functions $u(\cdot): T \rightarrow P$ and $v(\cdot): T \rightarrow E$ are called program controls of the partner and opponent, respectively. The sets of all program controls of the partner and opponent are denoted by the symbols $P_{T}(\cdot)$ and $E_{T}(\cdot): P_{T}(\cdot)=\left\{u(\cdot) \in L_{2}(T ; U): u(t) \in P\right.$ a.e. $\left.t \in T\right\}$, $E_{T}(\cdot)=\left\{v(\cdot) \in L_{2}(T ; V): v(t) \in E\right.$ a.e. $\left.t \in T\right\}$. Elements of the product $T \times \mathcal{H}$ are called positions, $\mathcal{H}=H \times H \times \mathbb{R} \times H \times H \times \mathbb{R}$. Any function (perhaps, multifunction) $\mathcal{U}: T \times \mathcal{H} \rightarrow P$ is said to be a positional strategy of the partner. The positional strategy corrects the controls at discrete times given by some partition of the interval $T$. Any function $\mathcal{V}: T \times H \times H \rightarrow H$ is said to be a strategy of reconstruction. The strategy $\mathcal{V}$ is formed in order to reconstruct the unknown component $\psi(\cdot)$.

Consider the following ordinary differential equation

$$
\dot{q}(t)=\chi(t, x(t), u(t)), \quad q\left(t_{0}\right)=0 .
$$

Introducing this new variable $q$, we reduce the control problem of Bolza type to a control problem with a terminal quality criterion of the form $I=\sigma(x(\vartheta))+$ 
$q(\vartheta)$. In this case, the controlled system consists of phase field equation (1) and ordinary differential equation (3).

The scheme of an algorithm for solving the problem undertaken by the partner is as follows. In the beginning, auxiliary systems $M_{1}$ and $M_{2}$ (models) are introduced. The system $M_{1}$ has an input $u^{*}(\cdot)$ and an output $w(\cdot)$; the system $M_{2}$, an input $p^{h}(\cdot)$ and an output $w_{*}(\cdot)$, respectively. The model $M_{2}$ with its control law $\mathcal{V}$ forms the identifier, whereas the model $M_{1}$ and system (1) (with their control laws) form the controller. The process of synchronous feedback control of systems (1), (3), $M_{1}$, and $M_{2}$ is organized on the interval $T$. This process is decomposed into $m-1$ identical steps. At the $i$ th step carried out during the time interval $\left[\tau_{i}, \tau_{i+1}\right)$, the following actions are fulfilled. First, at the time $\tau_{i}$, according to some chosen rules $\mathcal{V}$ and $\mathcal{U}$, the elements $p_{i}^{h} \in \mathcal{V}\left(\tau_{i}, \xi_{i}^{h}, w_{*}\left(\tau_{i}\right)\right), \quad u_{i}^{h} \in \mathcal{U}\left(\tau_{i}, \xi_{i}^{h}, p_{i}^{h}, \psi_{i}^{h}, w\left(\tau_{i}\right)\right)$ are calculated. Here, $\psi_{i}^{h}$ is the result of measuring $q\left(\tau_{i}\right)$. Then (till the moment $\tau_{i+1}$ ), the control $p^{h}(t)=p_{i}^{h}$, $\tau_{i} \leq t<\tau_{i+1}$, is fed onto the input of the system $M_{2}$; the control $u^{h}(t)=u_{i}^{h}$, $\tau_{i} \leq t<\tau_{i+1}$, onto the input of system (1), (3). Under the action of these controls, as well as of the given control $u^{*}(t), \tau_{i} \leq t<\tau_{i+1}$, and the unknown control of the opponent $v(t), \tau_{i} \leq t<\tau_{i+1}$, the states $x\left(\tau_{i+1}\right), q\left(\tau_{i}\right), w\left(\tau_{i+1}\right)$, and $w_{*}\left(\tau_{i+1}\right)$ are realized at the time $\tau_{i+1}$. The procedure stops at the time $\vartheta$.

Let models $M_{1}$ and $M_{2}$ with phase trajectories $w(\cdot)$ and $w_{*}(\cdot)$ be fixed. A solution $x(\cdot)$ of system (1) starting from an initial state $\left(t_{*}, x_{*}\right)$ and corresponding to piecewise constant controls $u^{h}(\cdot)$ and $p^{h}(\cdot)$ (formed by the feedback principle) and to a control $v_{t_{*}, \vartheta}(\cdot) \in E_{t_{*}, \vartheta}(\cdot)$ is called an $(h, \Delta)$-motion $x_{\Delta, w}^{h}(\cdot)=$ $x_{\Delta, w}^{h}\left(\cdot ; t_{*}, x_{*}, \mathcal{U}, \mathcal{V}, v_{t_{*}, \vartheta}(\cdot)\right)$. This motion is generated by the positional strategies $\mathcal{U}$ and $\mathcal{V}$. Thus, the motions $x_{\Delta, w}^{h}(\cdot), q_{\Delta}^{h}(\cdot), w(\cdot)$, and $w_{*}(\cdot)$ are formed simultaneously. So, for $t \in\left[\tau_{i}, \tau_{i+1}\right)$, we define $x_{\Delta, w}^{h}(t)=x\left(t ; \tau_{i}, x_{\Delta, w}^{h}\left(\tau_{i}\right), u_{\tau_{i}, \tau_{i+1}}^{h}(\cdot)\right.$, $\left.v_{\tau_{i}, \tau_{i+1}}(\cdot)\right), \quad q_{\Delta}^{h}(t)=q\left(t ; \tau_{i}, q_{\Delta}^{h}\left(\tau_{i}\right), u_{\tau_{i}, \tau_{i+1}}^{h}(\cdot)\right), w(t)=w\left(t ; \tau_{i}, w\left(\tau_{i}\right), u_{\tau_{i}, \tau_{i+1}}^{*}(\cdot)\right)$, $w_{*}(t)=w_{*}\left(t ; \tau_{i}, w_{*}\left(\tau_{i}\right), p_{\tau_{i}, \tau_{i+1}}^{h}(\cdot)\right)$, where

$$
\begin{gathered}
u^{h}(t)=u_{i}^{h} \in \mathcal{U}\left(\tau_{i}, \xi_{i}^{h}, p_{i}^{h}, q^{h}\left(\tau_{i}\right), w\left(\tau_{i}\right)\right), \quad p^{h}(t)=p_{i}^{h} \in \mathcal{V}\left(\tau_{i}, \xi_{i}^{h}, w_{*}\left(\tau_{i}\right)\right) \\
\quad \text { for } t \in\left[\tau_{i}, \tau_{i+1}\right), \\
i \in\left[i\left(t_{*}\right): m-1\right], \quad\left|\xi_{i}^{h}-\phi_{\Delta, w}^{h}\left(\tau_{i}\right)\right|_{H} \leq h, \quad\left|\psi_{i}^{h}-g_{\Delta}^{h}\left(\tau_{i}\right)\right| \leq h, \\
i\left(t_{*}\right)=\min \left\{i: \tau_{i}>t_{*}\right\}, \quad u^{h}(t)=u_{*}^{h} \in P, \quad p^{h}(t)=p_{*}^{h} \in H \quad \text { for } t \in\left[t_{*}, \tau_{i\left(t_{*}\right)}\right) .
\end{gathered}
$$

The set of all $(h, \Delta)$-motions is denoted by $X_{h}\left(t_{*}, x_{*}, \mathcal{U}, \mathcal{V}, \Delta, w\right)$.

Problem. It is necessary to construct a positional strategy $\mathcal{U}: T \times \mathcal{H} \rightarrow P$ of the partner and a positional strategy $\mathcal{V}: T \times H \times H \rightarrow H$ of reconstruction with the following properties: whatever a value $\varepsilon>0$ and a disturbance $v_{T}(\cdot) \in E_{T}(\cdot)$, one can find (explicitly) numbers $h_{*}>0$ and $\delta_{*}>0$ such that the inequalities

$$
\left|I\left(x_{\Delta, w}^{h}(\cdot), u_{T}^{h}(\cdot)\right)-I_{*}\right| \leq \varepsilon \quad \forall x_{\Delta, w}^{h}(\cdot) \in X_{h}\left(t_{0}, x_{0}, \mathcal{U}, \mathcal{V}, \Delta, w\right)
$$

are fulfilled uniformly with respect to all measurements $\xi_{i}^{h}$ and $\psi_{i}^{h}$ with properties (5), if $h \leq h_{*}$ and $\delta=\delta(\Delta) \leq \delta_{*}$. 


\section{Algorithm for solving the Problem}

To solve the Problem, we use ideas from [4], namely, the method of a priori stable sets. In our case, this method consists in the following. Let a trajectory of model $M_{1}, w(\cdot)$, possessing the property $\sigma\left(w_{1}(\vartheta)\right)+w_{2}(\vartheta)=I_{*}$ be known. Then, a feedback strategy $\mathcal{U}$ providing tracking the prescribed trajectory of $M_{1}$ by the trajectory of real system (1) is constructed. This means that the $(h, \Delta)$ motion $x_{\Delta}^{h}(\cdot)$ formed by the feedback principle (see (4)) remains at a "small" neighborhood of the trajectory $w(\cdot)$ during the whole interval $T$. This property of the $(h, \Delta)$-motion allows us to conclude that the chosen strategy solves the considered control problem.

Let us pass to the realization of this scheme. Define $\Phi(t, x, u, v)=\{B u-$ $C v, \chi(t, x, u)\} \in H \times \mathbb{R}, \Phi_{u}(t, x, v)=\bigcup_{u \in P} \Phi(t, x, u, v), H_{*}(t ; x)=\bigcap_{v \in E} \Phi_{u}(t, x, v)$, $H_{*}(\cdot ; x)=\left\{u(\cdot) \in L_{2}(T ; H \times \mathbb{R}): u(t) \in H_{*}(t ; x)\right.$ for $\quad$ a. $\left.a . t \in T\right\}$. As a model $M_{1}$, we take the system including two subsystems, i.e., the phase field equation

$$
\begin{gathered}
\frac{\partial}{\partial t} w^{(1)}+l \frac{\partial}{\partial t} w^{(2)}=\Delta_{L} w^{(1)}+u_{1} \quad \text { in } \quad \Omega \times\left(t_{0}, \vartheta\right], \\
\frac{\partial}{\partial t} w^{(2)}=\Delta_{L} w^{(2)}+g\left(w^{(2)}\right)+w^{(1)}
\end{gathered}
$$

with the boundary condition $\frac{\partial}{\partial n} w^{(1)}=\frac{\partial}{\partial n} w^{(2)}=0 \quad$ on $\quad \partial \Omega \times\left(t_{0}, \vartheta\right]$ and the initial condition $w^{(1)}\left(t_{0}\right)=\psi_{0}, \quad w^{(2)}\left(t_{0}\right)=\varphi_{0} \quad$ in $\Omega$, as well as the ordinary differential equation

$$
\dot{w}^{(3)}(t)=u_{2}(t), \quad \mathrm{w}^{(3)} \in \mathbb{R}, \quad \mathrm{w}^{(3)}(0)=0 .
$$

By the symbol $w(\cdot)$, we denote the solution of system (7), (8). Then, the model $M_{1}$ has the control $u(\cdot)=\left\{u_{1}(\cdot), u_{2}(\cdot)\right\}$. As a model $M_{2}$, we use the equation

$$
\frac{\partial}{w_{*}} \partial t=\Delta_{L} w_{*}+p^{h}+g\left(w_{*}\right) \quad \text { in } \quad \Omega \times\left(t_{0}, \vartheta\right]
$$

with the boundary condition $\frac{\partial w_{*}}{\partial n}=0 \quad$ on $\quad \partial \Omega \times\left(t_{0}, \vartheta\right]$ and the initial condition $w_{*}\left(t_{0}\right)=\varphi_{0} \quad$ in $\quad \Omega$.

Condition 1. There exists a control $u(\cdot)=u^{*}(\cdot)=\left\{u_{1}^{*}(\cdot), u_{2}^{*}(\cdot)\right\} \in H(t$; $\left.w^{(1)}(t), w^{(2)}(t)\right)$ for a.a. $t \in T$ such that $I_{*}=\sigma\left(w^{(1)}(\vartheta)\right)+w^{(2)}(\vartheta)$.

The strategies $\mathcal{U}$ and $\mathcal{V}$ (see (4)) are defined in such a way:

$$
\begin{gathered}
\mathcal{U}(t, \xi, p, \psi, w)=\arg \min \left\{L(u, y)+\left(\psi-w^{(3)}\right) \chi(t, \xi, p, u): u \in P\right\}, \\
\mathcal{V}\left(t, \xi, w_{*}\right)=\arg \min \left\{l(t, \alpha, u, s): u \in U_{d}\right\},
\end{gathered}
$$

where $w=\left\{w^{(1)}, w^{(2)}, w^{(3)}\right\}, L(u, y)=(-y, B u)_{H}, y=w^{(1)}-p+l\left(w^{(2)}-\xi\right)$, $l(t, \alpha, u, s)=\exp \left(-2 b_{*} t\right)(s, u)_{H}+\alpha|u|_{H}^{2}, s=w_{*}-\xi, b_{*}=|a+1 / 3 b|_{L_{\infty}(Q)}$, $\alpha=\alpha(h), U_{d}=\left\{p(\cdot) \in L_{2}(T ; H):|p(t)|_{H} \leq d\right.$ for a.a. $\left.t \in T\right\}, d \geq$ $\sup _{t \in T}\left\{\left|\psi\left(t ; t_{0}, x_{0}, u(\cdot), v(\cdot)\right)\right|_{H}: u(\cdot) \in P_{T}(\cdot), v(\cdot) \in E_{T}(\cdot)\right\}$. 
Condition 2. Let $h, \delta(h)$, and $\alpha(h)$ satisfy the conditions : $\alpha(h) \rightarrow 0, \delta(h) \rightarrow$ $0,(h+\delta(h)) \alpha^{-1}(h) \rightarrow 0$ as $h \rightarrow 0$.

Let us pass to the description of the algorithm for solving the Problem. Namely, we describe the procedure of forming the $(h, \Delta)$-motion $x_{\Delta, w}^{h}(\cdot)=$ $\left\{\psi_{\Delta, w}^{h}(\cdot), \varphi_{\Delta, w}^{h}(\cdot)\right\}$ and trajectory $g_{\Delta}^{h}(\cdot)$ corresponding to some fixed partition $\Delta$ and the strategies $\mathcal{U}$ and $\mathcal{V}$, see (10) and (11). Before the algorithm starts, we fix a value $h \in(0,1)$, a function $\alpha=\alpha(h):(0,1) \rightarrow(0,1)$, and a partition $\Delta_{h}=\left\{\tau_{h, i}\right\}_{i=0}^{m_{h}}$ with diameter $\delta=\delta(h)=\tau_{i+1}-\tau_{i}, \tau_{i}=\tau_{h, i}, \tau_{h, 0}=t_{0}, \tau_{h, m_{h}}=\vartheta$. The work of the algorithm is decomposed into $m_{h}-1$ identical steps. We assume that

$$
u^{h}(t)=u_{0}^{h} \in \mathcal{U}\left(t_{0}, \xi_{0}^{h}, p_{0}^{h}, 0, w\left(t_{0}\right)\right), \quad p^{h}(t)=p_{0}^{h} \in \mathcal{V}\left(t_{0}, \xi_{0}^{h}, \varphi_{0}\right)
$$

$\left(\left|\xi_{0}^{h}-\varphi_{0}\right|_{H} \leq h\right)$ on the interval $\left[t_{0}, \tau_{1}\right)$. Under the action of these piecewiseconstant controls as well as of an unknown disturbance $v_{t_{0}, \tau_{1}}(\cdot)$, the $(h, \Delta)$ motion $\left\{x_{\Delta, w}^{h}(\cdot)\right\}_{t_{0}, \tau_{1}}=\left\{\psi_{\Delta, w}^{h}\left(\cdot ; t_{0}, \psi_{0}, u_{t_{0}, \tau_{1}}^{h}(\cdot), v_{t_{0}, \tau_{1}}(\cdot)\right), \varphi_{\Delta, w}^{h}\left(\cdot, t_{0}, \varphi_{0}, u_{t_{0}, \tau_{1}}^{h}(\cdot)\right.\right.$, $\left.\left.v_{t_{0}, \tau_{t}}(\cdot)\right)\right\}_{t_{0}, \tau_{1}}$ of system (1), the trajectory $\left\{q_{\Delta}^{h}(\cdot)\right\}_{t_{0}, \tau}=\left\{q\left(\cdot ; t_{0}, q\left(t_{0}\right)\right)\right.$, $\left.\left.u_{t_{0}, \tau_{1}}^{h}(\cdot)\right)\right\}_{t_{0}, \tau_{1}}$ of equation (3), the trajectory $\left\{w_{*}(\cdot)\right\}_{t_{0}, \tau_{1}}=\left\{w_{*}\left(\cdot ; t_{0}, \phi_{0}\right.\right.$, $\left.\left.p_{t_{0}, \tau_{1}}^{h}(\cdot)\right)\right\}_{t_{0}, \tau_{1}}$ of the model $M_{2}$, and the trajectory $\{w(\cdot)\}_{t_{0}, \tau_{1}}=\left\{w\left(\cdot ; t_{0}, x_{0}\right.\right.$, $\left.\left.u_{t_{0}, \tau_{1}}^{*}(\cdot)\right)\right\}_{t_{0}, \tau_{1}}$ of the model $M_{1}$ are realized. At the time $t=\tau_{1}$, we determine $u_{1}^{h}$ and $p_{1}^{h}$ from the conditions

$$
u_{1}^{h} \in \mathcal{U}\left(\tau_{1}, \xi_{1}^{h}, p_{1}^{h}, \psi_{1}^{h}, w\left(\tau_{1}\right)\right), \quad p_{1}^{h} \in \mathcal{V}\left(\tau_{1}, \xi_{1}^{h}, w_{*}\left(\tau_{1}\right)\right)
$$

$\left(\left|\xi_{1}^{h}-\varphi_{\Delta, w}^{h}\left(\tau_{1}\right)\right|_{H} \leq h,\left|\psi_{1}^{h}-g_{\Delta}^{h}\left(\tau_{1}\right)\right| \leq h\right)$, i.e., we assume that $u^{h}(t)=u_{1}^{h}$ and $p^{h}(t)=p_{1}^{h}$ for $t \in\left[\tau_{1}, \tau_{2}\right)$. Then, we calculate the realization of the $(h, \Delta)$-motion

$$
\begin{aligned}
\left\{x_{\Delta, w}^{h}(\cdot)\right\}_{\tau_{1}, \tau_{2}}= & \left\{\psi_{\Delta, w}^{h}\left(\cdot ; \tau_{1}, \psi_{\Delta, w}^{h}\left(\tau_{1}\right), u_{\tau_{1}, \tau_{2}}^{h}(\cdot), v_{\tau_{1}, \tau_{2}}(\cdot)\right),\right. \\
& \left.\varphi_{\Delta, w}^{h}\left(\cdot ; \tau_{1}, \varphi_{\Delta, w}^{h}\left(\tau_{1}\right), u_{\tau_{1}, \tau_{2}}^{h}(\cdot), v_{\tau_{1}, \tau_{2}}(\cdot)\right)\right\}_{\tau_{1}, \tau_{2}},
\end{aligned}
$$

the trajectory $\left\{q_{\Delta}^{h}(\cdot)\right\}_{\tau_{1}, \tau_{2}}=\left\{q\left(\cdot ; \tau_{1}, q_{\Delta}^{h}\left(\tau_{1}\right), p_{\tau_{1}, \tau_{2}}^{h}(\cdot)\right)\right\}_{\tau_{1}, \tau_{2}}$ of equation (3), the trajectory $\left\{w_{*}(\cdot)\right\}_{\tau_{1}, \tau_{2}}=\left\{w_{*}\left(\cdot ; \tau_{1}, w_{*}\left(\tau_{1}\right), p_{\tau_{1}, \tau_{2}}^{h}(\cdot)\right)\right\}_{\tau_{1}, \tau_{2}}$ of the model $M_{2}$, and the trajectory $\{w(\cdot)\}_{\tau_{1}, \tau_{2}}=\left\{w\left(\cdot ; \tau_{1}, w\left(\tau_{1}\right), u_{\tau_{1}, \tau_{2}}^{*}(\cdot)\right\}_{\tau_{1}, \tau_{2}}\right.$ of the model $M_{1}$.

Let the $(h, \Delta)$-motion $x_{\Delta, w}^{h}(\cdot)$, the trajectory $q_{\Delta}^{h}(\cdot)$ of equation (3), the trajectory $w_{*}(\cdot)$ of the model $M_{2}$, and the trajectory $w(\cdot)$ of the model $M_{1}$ be defined on the interval $\left[t_{0}, \tau_{i}\right]$. At the time $t=\tau_{i}$, we assume that

$$
u_{i}^{h} \in \mathcal{U}\left(\tau_{i}, \xi_{i}^{h}, p_{i}^{h}, \psi_{i}^{h}, w\left(\tau_{i}\right)\right), \quad p_{i}^{h} \in \mathcal{V}\left(\tau_{i}, \xi_{i}^{h}, w_{*}\left(\tau_{i}\right)\right)
$$

$\left(\left|\xi_{i}^{h}-\varphi_{\Delta, w}^{h}\left(\tau_{i}\right)\right|_{H} \leq h,\left|\psi_{i}^{h}-g_{\Delta}^{h}\left(\tau_{i}\right)\right| \leq h\right)$, i.e., we set $u^{h}(t)=u_{i}^{h} \quad$ and $\quad p^{h}(t)=$ $p_{i}^{h}$ for $t \in\left[\tau_{i}, \tau_{i+1}\right)$. As a result of the action of these controls and an unknown disturbance $v_{\tau_{i}, \tau_{i+1}}(\cdot)$, the $(h, \Delta)$-motion

$$
\begin{aligned}
\left\{x_{\Delta, w}^{h}(\cdot)\right\}_{\tau_{i}, \tau_{i+1}}= & \left\{\psi_{\Delta, w}^{h}\left(\cdot ; \tau_{i}, \psi_{\Delta, w}^{h}\left(\tau_{i}\right), u_{\tau_{i}, \tau_{i+1}}^{h}(\cdot), v_{\tau_{i}, \tau_{i+1}}(\cdot)\right),\right. \\
& \left.\varphi\left(\cdot ; \tau_{i}, \varphi_{\Delta, w}^{h}\left(\tau_{i}\right), u_{\tau_{i}, \tau_{i+1}}^{h}(\cdot), v_{\tau_{i}, \tau_{i+1}}(\cdot)\right)\right\}_{\tau_{i}, \tau_{i+1}},
\end{aligned}
$$

the trajectory $\left\{q_{\Delta}^{h}(\cdot)\right\}_{\tau_{i}, \tau_{i+1}}=\left\{q\left(\cdot ; \tau_{i}, q_{\Delta}^{h}\left(\tau_{i}\right), p_{\tau_{i}, \tau_{i+1}}^{h}(\cdot)\right\}_{\tau_{i}, \tau_{i+1}}\right.$ of equation (3), the trajectory $\left\{w_{*}(\cdot)\right\}_{\tau_{i}, \tau_{i+1}}=\left\{w_{*}\left(\cdot ; \tau_{i}, w_{*}\left(\tau_{i}\right), p_{\tau_{i}, \tau_{1+1}}^{h}(\cdot)\right)\right\}_{\tau_{i}, \tau_{i+1}}$ of the model 
$M_{2}$, and the trajectory $\{w(\cdot)\}_{\tau_{i}, \tau_{i+1}}=\left\{w\left(\cdot ; \tau_{i}, w\left(\tau_{i}\right), u_{\tau_{i}, \tau_{i+1}}^{*}(\cdot)\right)\right\}_{\tau_{i}, \tau_{i+1}}$ of the model $M_{1}$ are realized on the interval $\left[\tau_{i}, \tau_{i+1}\right]$. This procedure stops at the time $\vartheta$.

Theorem 2. Let Conditions 1 and 2 be fulfilled. Let also the models $M_{1}$ and $M_{2}$ be specified by relations (7), (8), and (9), respectively. Then, the strategies $\mathcal{U}$ and $\mathcal{V}$ of form (10) and (11) solve the Problem.

Proof. To prove the theorem, we estimate the variation of the functional

$$
\begin{aligned}
& \Lambda\left(t, x_{\Delta, w}^{h}(\cdot), q_{\Delta}^{h}(\cdot), w(\cdot)\right)=\Lambda^{0}\left(x_{\Delta, w}^{h}(t), q_{\Delta}^{h}(t), w(t)\right)+ \\
& +0.5 \int_{0}^{t}\left\{\int_{\Omega}\left|\nabla \pi^{h}(\varrho, \eta)\right|^{2} \mathrm{~d} \eta+l^{2} \int_{\Omega}\left|\nabla \nu^{h}(\varrho, \eta)\right|^{2} \mathrm{~d} \eta\right\} \mathrm{d} \varrho,
\end{aligned}
$$

where $x_{\Delta, w}^{h}(\cdot)=\left\{\psi_{\Delta, w}^{h}(\cdot), \phi_{\Delta, w}^{h}(\cdot)\right\}, w(\cdot)=\left\{w^{(1)}(\cdot), w^{(2)}(\cdot), w^{(3)}(\cdot)\right\}, \quad \pi^{h}(t)=$ $w^{(1)}(t)-\psi_{\Delta, w}^{h}(t), \nu^{h}(t)=w^{(2)}(t)-\varphi_{\Delta, w}^{h}(t), g^{h}(t)=\pi^{h}(t)+l \nu^{h}(t), \Lambda_{1}\left(x_{\Delta, w}^{h}(t)\right.$, $w(t))=0.5\left|g^{h}(t)\right|_{H}^{2}+0.5 l^{2}\left|\nu^{h}(t)\right|_{H}^{2}, \quad \lambda\left(q_{\Delta}^{h}(t), w^{(3)}(t)\right)=0.5\left|q_{\Delta}^{h}(t)-w^{(3)}(t)\right|^{2}$, $\Lambda^{0}\left(x_{\Delta, w}^{h}(t), q_{\Delta}^{h}(t), w(t)\right)=\Lambda_{1}\left(x_{\Delta, w}^{h}(t), w(t)\right)+\lambda\left(q_{\Delta}^{h}(t), w^{(3)}(t)\right.$. It is easily seen that the functions $\pi^{h}(\cdot)$ and $\nu^{h}(\cdot)$ are solutions of the system

$$
\begin{gathered}
\frac{\partial \pi^{h}(t, \eta)}{\partial t}+l \frac{\partial \nu^{h}(t, \eta)}{\partial t}=\Delta_{L} \pi^{h}(t, \eta)+u_{1}^{*}(t, \eta)-\left(B u^{h}\right)(t, \eta)+(C v)(t, \eta), \\
\frac{\partial \nu^{h}(t, \eta)}{\partial t}=\Delta_{L} \nu^{h}(t, \eta)+R^{h}(t, \eta) \nu^{h}(t, \eta)+\pi^{h}(t, \eta) \quad \text { in } \Omega \times\left(t_{0}, \vartheta\right],
\end{gathered}
$$

with the initial condition $\pi^{h}\left(t_{0}\right)=\nu^{h}\left(t_{0}\right)=0$ in $\Omega$ and with the boundary condition $\frac{\partial \pi^{h}}{\partial n}=\frac{\partial \nu^{h}}{\partial n}=0$ on $\partial \Omega \times\left(t_{0}, \vartheta\right]$. Here, $R^{h}(t, \eta)=a(t, \eta)+b(t, \eta)\left(w^{(1)}(t, \eta)+\right.$ $\left.\varphi_{\Delta, w}^{h}(t, \eta)\right)-\left(\left(w^{(1)}(t, \eta)\right)^{2}+w^{(1)}(t, \eta) \varphi_{\Delta, w}^{h}(t, \eta)+\left(\varphi_{\Delta, w}^{h}\right)^{2}(t, \eta)\right)$. Multiplying scalarly the first equation of (13) by $g^{h}(t)$, and the second one, by $\nu^{h}(t)$, we obtain

$$
\begin{gathered}
\left(g^{h}(t), g_{t}^{h}(t)\right)_{H}+\int_{\Omega}\left\{\left|\nabla \pi^{h}(t, \eta)\right|^{2}+l \nabla \pi^{h}(t, \eta) \nabla \nu^{h}(t, \eta)\right\} \mathrm{d} \eta= \\
=\left(g^{h}(t), u_{1}^{*}(t)-B u^{h}(t)+C v(t)\right)_{H}, \\
\left(\nu^{h}(t), \nu_{t}^{h}(t)\right)_{H}+\int_{\Omega}\left|\nabla \nu^{h}(t, \eta)\right|^{2} \mathrm{~d} \eta \leq\left(\pi^{h}(t), \nu^{h}(t)\right)_{H}+b\left|\nu^{h}(t)\right|_{H}^{2} \quad \text { for a.a. } t \in T .
\end{gathered}
$$

Here, we use the inequality $\underset{(t, \eta) \in T \times \Omega}{\operatorname{vraimax}}\left\{a(t, \eta)+b(t, \eta)\left(v_{1}+v_{2}\right)-\left(v_{1}^{2}+v_{1} v_{2}+v_{2}^{2}\right)\right\} \leq$ $b$, which is valid for any $v_{1}, v_{2} \in \mathbb{R}$. It is evident that the inequality

$$
\int_{\Omega} l\left(\nabla \pi^{h}(t, \eta), \nabla \nu^{h}(t, \eta)\right) \mathrm{d} \eta \geq-0.5 \int_{\Omega}\left\{\left|\nabla \pi^{h}(t, \eta)\right|^{2}+l^{2}\left|\nabla \nu^{h}(t, \eta)\right|^{2}\right\} \mathrm{d} \eta
$$


is fulfilled for a.a. $t \in T$. Let us multiply the first inequality of (14) by $l^{2}$ and add to the second one. Taking into account (15), we have for a.a. $t \in T$

$$
\begin{gathered}
\left(g^{h}(t), g_{t}^{h}(t)\right)_{H}+l^{2}\left(\nu^{h}(t), \nu_{t}^{h}(t)\right)_{H}+0.5 \int_{\Omega}\left\{\left|\nabla \pi^{h}(t, \eta)\right|^{2}+l^{2}\left|\nabla \nu^{h}(t, \eta)\right|^{2}\right\} \mathrm{d} \eta \text { (16) } \\
\leq\left(g^{h}(t), u_{1}^{*}(t)-B u^{h}(t)+C v(t)\right)_{H}+l^{2}\left(\pi^{h}(t), \nu^{h}(t)\right)_{H}+b l^{2}\left|\nu^{h}(t)\right|_{H}^{2} .
\end{gathered}
$$

Note that $\pi^{h}(t)=g^{h}(t)-l \nu^{h}(t)$. In this case, for a.a. $t \in T$

$$
\begin{aligned}
& \left(\pi^{h}(t), \nu^{h}(t)\right)_{H}+b\left|\nu^{h}(t)\right|_{H}^{2}=\left(g^{h}(t)-l \nu^{h}(t), \nu^{h}(t)\right)_{H}+b\left|\nu^{h}(t)\right|_{H}^{2}= \\
= & \left(g^{h}(t), \nu^{h}(t)\right)_{H}+(b-l)\left|\nu^{h}(t)\right|_{H}^{2} \leq 0.5\left(\left|g^{h}(t)\right|_{H}^{2}+(0.5+|b-l|)\left|\nu^{h}(t)\right|_{H}^{2} .\right.
\end{aligned}
$$

Combining (16) and (17), we obtain

$$
\begin{gathered}
\frac{\mathrm{d}}{\mathrm{d} t} \Lambda^{0}\left(x_{\Delta, w}^{h}(t), q_{\Delta}^{h}(t), w(t)\right)+0.5 \int_{\Omega}\left\{\left|\nabla \pi^{h}(t, \eta)\right|^{2}+l^{2}\left|\nabla \nu^{h}(t, \eta)\right|^{2}\right\} \mathrm{d} \eta \leq \\
\leq 2 l^{2} \lambda^{2} \Lambda^{0}\left(x_{\Delta, w}^{h}(t), q_{\Delta}^{h}(t), w(t)\right)+\gamma_{t}^{(1)}+\gamma_{t}^{(2)},
\end{gathered}
$$

where $\gamma_{t}^{(1)}=\left(q_{\Delta}^{h}(t)-w^{(3)}(t)\right)\left(\chi\left(t, x_{\Delta, w}^{h}(t), u^{h}(t)\right)-u_{2}^{*}(t)\right), \gamma_{t}^{(2)}=\left(g^{h}(t), u_{1}^{*}(t)-\right.$ $\left.B u^{h}(t)+C v(t)\right)_{H}$ for a.a. $t \in T$. For a.a. $t \in \delta_{i}=\left[\tau_{i}, \tau_{i+1}\right]$, it is easily seen that

$$
\begin{gathered}
\gamma_{t}^{(1)} \leq\left(q_{\Delta}^{h}\left(\tau_{i}\right)-w^{(3)}\left(\tau_{i}\right)\right)\left(\chi\left(t, x_{\Delta, w}^{h}(t), u^{h}(t)-u_{2}^{*}(t)\right)+k_{0}\left(t-\tau_{i}\right)^{1 / 2}, \quad(19)\right. \\
\left|\chi\left(t, x_{\Delta, w}^{h}(t), u^{h}(t)\right)-\chi\left(\tau_{i}, \xi_{i}^{h}, p_{i}^{h}, u^{h}(t)\right)\right| \leq k_{1}\left\{h+\left(t-\tau_{i}\right)^{1 / 2}+\left|p_{i}^{h}-\psi_{\Delta, w}^{h}(t)\right|_{H}^{2}\right\} .
\end{gathered}
$$

From (19) and (20) we have for a.a. $t \in \delta_{i}$

$$
\begin{gathered}
\gamma_{t}^{(1)} \leq\left(q_{\Delta}^{h}\left(\tau_{i}\right)-w^{(3)}\left(\tau_{i}\right)\right)\left(\chi\left(\tau_{i}, \xi_{i}^{h}, \psi_{i}^{h}, u^{h}(t)\right)-u_{2}^{*}(t)\right)+ \\
+k_{2}\left|q_{\Delta}^{h}\left(\tau_{i}\right)-w^{(3)}\left(\tau_{i}\right)\right|\left\{h+\left(t-\tau_{i}\right)^{1 / 2}+\left|p_{i}^{h}-\psi_{\Delta, w}^{h}(t)\right|_{H}\right\} .
\end{gathered}
$$

Estimate the last term in the right-hand side of inequality (18). For a.a. $t \in$ $\left[\tau_{i}, \tau_{i+1}[\right.$

$$
\left|g^{h}(t)-y_{i}^{h}\right|_{H}=\left|\pi^{h}(t)+l \nu^{h}(t)-y_{i}^{h}\right|_{H} \leq \lambda_{1, i}(t)+\lambda_{2, i}(t),
$$

where $y_{i}^{h}=w^{(1)}\left(\tau_{i}\right)-p_{i}^{h}-l\left(w^{(2)}\left(\tau_{i}\right)-\xi_{i}^{h}\right), \lambda_{1, i}(t)=\mid w^{(1)}(t)-\psi_{\Delta, w}^{h}(t)-w^{(1)}\left(\tau_{i}\right)+$ $\left.p_{i}^{h}\right|_{H}, \lambda_{2, i}(t)=l\left|w^{(2)}(t)-\varphi_{\Delta, w}^{h}(t)-w^{(2)}\left(\tau_{i}\right)+\xi_{i}^{h}\right|_{H}$. For a.a. $t \in \delta_{i}=\left[\tau_{i}, \tau_{i+1}\right]$, it is easily seen that $\lambda_{1, i}(t) \leq\left|p_{i}^{h}-\psi_{\Delta, w}^{h}(t)\right|_{H}+\int_{\tau_{i}}^{t}\left|\dot{w}^{(1)}(\tau)\right|_{H} \mathrm{~d} \tau, \lambda_{2, i}(t) \leq l h+$ $l \int_{\tau_{i}}^{t}\left\{\left|\dot{\varphi}_{\Delta, w}^{h}(\tau)\right|_{H}+\left|\dot{w}^{(2)}(\tau)\right|_{H}\right\} \mathrm{d} \tau$. From this equation and (22), for a.a. $t \in \delta_{i}$, it follows that

$$
\left|g^{h}(t)-y_{i}^{h}\right|_{H} \leq l h+\int_{\tau_{i}}^{t}\left\{l\left|\dot{\varphi}_{\Delta, w}^{h}(\tau)\right|_{H}+\left|\dot{w}^{(1)}(\tau)\right|_{H}+\left|\dot{w}^{(2)}(\tau)\right|_{H}\right\} \mathrm{d} \tau+\left|p_{i}^{h}-\psi_{\Delta, w}^{h}(t)\right|_{H} .
$$


By virtue of (11), taking into account results of $[8,9]$, from estimate (23) we derive

$$
\sum_{i=0}^{m-1} \int_{\tau_{i}}^{\tau_{i}+1} M\left(t ; \tau_{i}\right) \mathrm{d} t \leq k_{3}(h+\delta)+k_{4} \int_{t_{0}}^{\vartheta}\left|p^{h}(\tau)-\psi_{\Delta, w}^{h}(\tau)\right|_{H} \mathrm{~d} \tau \leq k_{5} \nu^{1 / 2}(h),
$$

where $M\left(t ; \tau_{i}\right)=\left|g^{h}(t)-y_{i}^{h}\right|_{H}\left\{\left|B u_{i}^{h}\right|_{H}+|C v(t)|_{H}+\left|u_{1}^{*}(t)\right|_{H}\right\}$ for a.a. $t \in \delta_{i}$, $\nu(h)=(h+\delta(h)+\alpha(h))^{1 / 2}+(h+\delta(h)) \alpha^{-1}(h)$. Note that, for a.a. $t \in \delta_{i}$,

$$
\gamma_{t}^{(2)} \leq\left(y_{i}^{h}, u_{1}^{*}(t)-B u_{i}^{h}+C v(t)\right)_{H}+M\left(t ; \tau_{i}\right) .
$$

Let the symbol $(\cdot, \cdot)_{H \times \mathbb{R}}$ denote the scalar product in the space $H \times \mathbb{R}$. Let us define elements $v_{i}^{e}$ from the conditions

$$
\inf _{u \in P}\left(s_{i}, \Phi\left(\tau_{i}, p_{i}^{h}, \xi_{i}^{h}, u, v_{i}^{e}\right)\right)_{H \times \mathbb{R}} \geq \sup _{v \in E} \inf _{u \in P}\left(s_{i}, \Phi\left(\tau_{i}, \xi_{i}^{h}, u, v\right)\right)_{H \times \mathbb{R}}-h,
$$

where $\Phi\left(\tau_{i}, p_{i}^{h}, \xi_{i}^{h}, u_{i}^{h}, v(t)\right)=\left\{B u_{i}^{h}-C v(t), \chi\left(\tau_{i}, \xi_{i}^{h}, p_{i}^{h}, u_{i}^{h}\right)\right\}, s_{i}=\left\{-y_{i}^{h}, \psi_{i}^{h}-\right.$ $\left.w^{(3)}\left(\tau_{i}\right)\right\}$. It is obvious (see Condition 1) that $u_{*}(t) \in H\left(t, w^{(1)}(t), w^{(2)}(t)\right) \subset$ $\bigcup_{u \in P} \Phi\left(t, w^{(1)}(t), w^{(2)}(t), u, v_{i}^{e}\right)$ for a. a. $t \in\left[\tau_{i}, \tau_{i+1}\right)$. Then, for a.a. $t \in \delta_{i}$, there exists a control $u^{(1)}(t) \in P$ such that

$$
\Phi\left(t, w^{(1)}(t), w^{(2)}(t), u^{(1)}(t), v_{i}^{e}\right)=u^{*}(t) \quad \text { for a. a. } \quad t \in\left[\tau_{i}, \tau_{i+1}\right] .
$$

Using the rule of definition of the strategy $\mathcal{U}$, we deduce that

$$
\left(s_{i}, \Phi\left(\tau_{i}, p_{i}^{h}, \xi_{i}^{h}, u_{i}^{h}, v(t)\right)\right)_{H \times \mathbb{R}} \leq \inf _{u \in P} \sup _{v \in E}\left(s_{i}, \Phi\left(\tau_{i}, p_{i}^{h}, \xi_{i}^{h}, u, v\right)\right)_{H \times \mathbb{R}}+h .
$$

Here (see (12)), $u_{i}^{h} \in \mathcal{U}\left(\tau_{i}, \xi_{i}^{h}, p_{i}^{h}, \psi_{i}^{h}, w\left(\tau_{i}\right)\right) ; v_{\tau_{i}, \tau_{i+1}}(\cdot)$ is an unknown realization of the control of the opponent. In turn, from (26) we have

$$
\sup _{v \in E} \inf _{u \in P}\left(s_{i}, \Phi\left(\tau_{i}, p_{i}^{h}, \xi_{i}^{h}, u, v\right)\right)_{H \times \mathbb{R}} \leq \inf _{u \in P}\left(s_{i}, \Phi\left(\tau_{i}, p_{i}^{h}, \xi_{i}^{h}, u, v_{i}^{e}\right)\right)_{H \times \mathbb{R}}+h .
$$

Moreover, it is evident that the equality

$$
\inf _{u \in P} \sup _{v \in E}\left(s_{i}, \Phi\left(\tau_{i}, p_{i}^{h}, \xi_{i}^{h}, u, v\right)\right)_{H \times \mathbb{R}}=\sup _{v \in E} \inf _{u \in P}\left(s_{i}, \Phi\left(\tau_{i}, p_{i}^{h}, \xi_{i}^{h}, u, v\right)\right)_{H \times \mathbb{R}}
$$

is valid. From (28)-(30) we have

$$
\begin{array}{r}
\left(s_{i}, \Phi\left(\tau_{i}, p_{i}^{h}, \xi_{i}^{h}, u_{i}^{h}, v(t)\right)\right)_{H \times \mathbb{R}} \leq \inf _{u \in P}\left(s_{i}, \Phi\left(\tau_{i}, p_{i}^{h}, \xi_{i}^{h}, u, v_{i}^{e}\right)\right)_{H \times \mathbb{R}}+2 h \leq \\
\leq\left(s_{i}, \Phi\left(t, p_{i}^{h}, \xi_{i}^{h}, u^{(1)}(t), v_{i}^{e}\right)\right)_{H \times \mathbb{R}}+2 h+L\left|\psi_{i}^{h}-w^{(3)}\left(\tau_{i}\right)\right|\left(t-\tau_{i}\right) .
\end{array}
$$

Here, $L$ is the Lipschitz constant of the function $\chi(\cdot)$ in the corresponding domain. In this case, for a.a. $t \in \delta_{i}$, it follows from (27), (31) that

$$
\left(s_{i}, \Phi\left(\tau_{i}, p_{i}^{h}, \xi_{i}^{h}, u_{i}^{h}, v(t)\right)-u^{*}(t)\right)_{H \times \mathbb{R}} \leq
$$




$$
\leq 2 h+L\left|\psi_{i}^{h}-w^{(3)}\left(\tau_{i}\right)\right|\left\{t-\tau_{i}+\left|\xi_{i}^{h}-w^{(1)}(t)\right|_{H}+\left|p_{i}^{h}-w^{(2)}(t)\right|_{H}\right\} .
$$

By virtue of (32) and (21), for the interval $\left[\tau_{i}, \tau_{i+1}\right]$, we derive

$$
\begin{gathered}
\gamma_{t}^{(1)}+\gamma_{t}^{(2)} \leq \\
\leq \pi_{i}^{*}(t)+k_{6}\left\{h^{2}+t-\tau_{i}+\Lambda^{0}\left(x_{\Delta, w}^{h}(t), q_{\Delta}^{h}(t), w(t)\right)+\left|p_{i}^{h}-\psi_{\Delta, w}^{h}(t)\right|_{H}^{2}\right\},
\end{gathered}
$$

where

$$
\pi_{i}^{*}(t)=\left(s_{i}, \Phi\left(\tau_{i}, p_{i}^{h}, \xi_{i}^{h}, u_{i}^{h}, v(t)\right)-u^{*}(t)\right)_{H \times \mathbb{R}} .
$$

We deduce from inequalities (18) and (33) that

$$
\frac{\mathrm{d} \Lambda\left(t, x_{\Delta, w}^{h}(\cdot), q_{\Delta}^{h}(\cdot), w(\cdot)\right)}{\mathrm{d} t} \leq M\left(t ; \tau_{i}\right)+
$$

$+k_{6} \Lambda\left(t, x_{\Delta, w}^{h}(\cdot), q_{\Delta}^{h}(\cdot), w(\cdot)\right)+k_{7}\left\{h^{2}+t-\tau_{i}+\left|p_{i}^{h}-\psi_{\Delta, w}^{h}(t)\right|_{H}^{2}\right\} \quad$ for a.a. $t \in \delta_{i}$.

Using (34) and (24), by virtue of the Gronwall lemma, we obtain for $t \in T$

$$
\Lambda\left(t, x_{\Delta, w}^{h}(\cdot), q_{\Delta, w}^{h}(\cdot), w(\cdot)\right) \leq k_{8} \sum_{i=0}^{m-1}\left\{\int_{\tau_{i}}^{\tau_{i+1}} M\left(t ; \tau_{i}\right) \mathrm{d} t+\delta\left(h^{2}+\delta\right)\right\} \leq k_{9} \nu^{1 / 2}(h) .
$$

The statement of the theorem follows from the last inequality.

\section{References}

1. Fattorini, H.O.: Infinite Dimensional Optimization and Control Theory. Cambridge University Press, Cambridge (1999)

2. Lasiecka, I., Triggiani, R.: Control theory for partial differential equations: continuous and approximation theories. Part I: Abstract parabolic systems. Part II: Abstract hyperbolic-like systems over a finite time horizon. Cambridge University press, Cambridge (2000).

3. Tröltzsch, F.: Optimal Control of Partial Differential Equations. Theory, Methods and Applications. AMS, Providence, Rhode Island (2010)

4. Krasovskii, N., Subbotin, A.: Game-Theoretical Control Problems, Springer, Berlin (1988)

5. Caginalp, G.: An Analysis of a Phase Field Model of a Free Boundary. Arch. Rat. Mech. Analysis. 92, 205-245 (1986)

6. Hoffman, K.-H., Jiang, L.S.: Optimal Control Problem of a Phase Field Model for Solidification. Numer. Funct. Anal. and Optimiz. 13(1-2), 11-27 (1992)

7. Maksimov, V.I.: Dynamical Inverse Problems of Distributed Systems. VSP, Boston (2002)

8. Maksimov, V., Tröltzsch, F.: On an Inverse Problem for Phase Field Equations. Dokl. Akad. Nauk. 396(3), 309-312 (2004)

9. Maksimov, V., Tröltzsch, F.: Dynamical State and Control Reconstruction for a Phase Field Model. Dynamics of Continuous, Discrete and Impulsive Systems. A: Mathematical analysis. 13(3-4), 419-444 (2006)

10. Liu, Sh., Triggiani, R.: Inverse problem for a linearized Jordan-Moore-GibsonThompson equation. In book: New Prospects in Direct, Inverse and Control Problems for Evolution Equations, Springer, 305-352 (2014) 